\title{
ALFABURST:
}

\section{A realtime fast radio burst monitor for the Arecibo telescope}

\author{
Jayanth Chennamangalam*1, Aris Karastergiou ${ }^{1,2,3}$, David MacMahon ${ }^{4}$, Wes Armour ${ }^{5}$, Jeff \\ $\mathrm{Cobb}^{4}$, Duncan Lorimer ${ }^{6,7}$, Kaustubh Rajwade ${ }^{6}$, Andrew Siemion ${ }^{4,8,9}$, Dan Werthimer ${ }^{4}$, \\ Christopher Williams ${ }^{1}$ \\ ${ }^{1}$ Astrophysics, University of Oxford, \\ Denys Wilkinson Building, Keble Road, Oxford OX1 3RH, UK \\ *E-mail: jayanth@astro.ox.ac.uk \\ www-astro.physics.ox.ac.uk \\ ${ }^{2}$ Department of Physics and Electronics, Rhodes University, \\ PO Box 94, Grahamstown 6140, South Africa \\ ${ }^{3}$ Physics Department, University of the Western Cape, \\ Cape Town 7535, South Africa \\ ${ }^{4}$ Department of Astronomy, University of California Berkeley, \\ Berkeley, CA 94720, USA \\ ${ }^{5}$ Oxford e-Research Centre, University of Oxford, \\ Keble Road, Oxford OX1 3QG, UK \\ ${ }^{6}$ Department of Physics and Astronomy, West Virginia University, \\ PO Box 6315, Morgantown, WV 26506, USA \\ ${ }^{7}$ National Radio Astronomy Observatory, \\ PO Box 2, Green Bank, WV 24944, USA \\ ${ }^{8}$ ASTRON, PO Box 2, 7990 AA Dwingeloo, The Netherlands \\ ${ }^{9}$ Department of Astrophysics, Radboud University, \\ PO Box 9010, 6500 GL Nijmegen, The Netherlands
}

Fast radio bursts (FRBs) constitute an emerging class of fast radio transient whose origin continues to be a mystery. Realizing the importance of increasing coverage of the search parameter space, we have designed, built, and deployed a realtime monitor for FRBs at the 305-m Arecibo radio telescope. Named 'ALFABURST', it is a commensal instrument that is triggered whenever the $1.4 \mathrm{GHz}$ seven-beam Arecibo $L$-Band Feed Array (ALFA) receiver commences operation. The ongoing commensal survey we are conducting using ALFABURST has an instantaneous field of view of $0.02 \mathrm{sq}$. deg. within the FWHM of the beams, with the realtime software configurable to use up to $300 \mathrm{MHz}$ of bandwidth. We search for FRBs with dispersion measure up to $2560 \mathrm{~cm}^{-3}$ pc and pulse widths ranging from $0.128 \mathrm{~ms}$ to $16.384 \mathrm{~ms}$. Commissioning observations performed over the past few months have demonstrated the capability of the instrument in detecting single pulses from known pulsars. In this paper, I describe the instrument and the associated survey.

Keywords: instrumentation; fast radio bursts; pulsars 


\section{Introduction}

Fast radio bursts (FRBs) are non-repeating, millisecond-duration, broadband, dispersed radio pulses that exhibit values of dispersion measure (DM) much larger than what is expected due to the Galactic electron density ${ }^{6112}$ This latter fact prima facie leads to the inference that they are extragalactic in origin, with the millisecond-duration pulse-widths implying compact object progenitors. Although a few Galactic-origin hypotheses have been put forward (see, for example, Ref. 5), most theories of FRBs present cosmological explanations, including, for example, the collapse of supramassive neutron stars to black holes, ${ }^{3}$ and Alfvén wings around planets orbiting extragalactic pulsars. ${ }^{8}$ Only a few FRBs are known, and all except one were discovered using the Parkes telescope. The exception was discovered using the Arecibo telescope. ${ }^{111}$ If FRBs are indeed extragalactic in origin, they can be used as probes of the intergalactic environment, for example, to measure the intergalactic baryon content, helping solve the long-standing 'missing baryon problem' in cosmology, wherein a discrepancy exists between the expected and observed quantities of baryons in the Universe. $\frac{799}{70 w e v e r, ~ d u e ~ t o ~ t h e ~ l a r g e ~ u n c e r t a i n t y ~ i n ~ l o c a l i z a t i o n ~}$ of these bursts, a conclusive explanation of their origin is still lacking. Recent efforts have focused on automated, realtime searches for FRBs to try to increase the sample size, $\stackrel{4110}{=}$ and, in case of detection, perform signal characterization. 10

We have recently designed, developed, and deployed one such realtime FRB search pipeline for the 305-m diameter Arecibo telescope. Named 'ALFABURST', it is a commensal data processing system that utilizes the $1.4 \mathrm{GHz}$ seven-beam Arecibo $L$-band Feed Array (ALFA) receiver. ALFA is Arecibo's workhorse survey receiver, being used in the successful Pulsar ALFA (PALFA) survey, ${ }^{2}$ that has so far resulted in the discovery of 145 pulsars, and one FRB. ALFABURST piggybacks on the commensal data processing infrastructure developed for the SERENDIP VI system, the latest iteration of the SERENDIP series of SETI spectrometers at Arecibo. It also partially re-uses ARTEMIS, the FRB search code developed for the recently concluded ARTEMIS survey at the UK station of the LOFAR telescope ${ }^{4}$. A detailed description of the instrument is given in Sec. 2 .

For the commensal survey that we plan to conduct, the constituent observations are triggered automatically whenever the primary observer enables the ALFA receiver. Based on historical and expected usage of ALFA, we expect to observe for $500-1000$ hours over the next year. A detailed description of the survey is give in Sec. 3 ,

\section{System Description}

ALFABURST is a heterogeneous instrument, i.e., it combines two Field Programmable Gate Array (FPGA) boards - namely, the ROACH2, developed by the CASPER collaboration - with a high-performance computing (HPC) cluster 


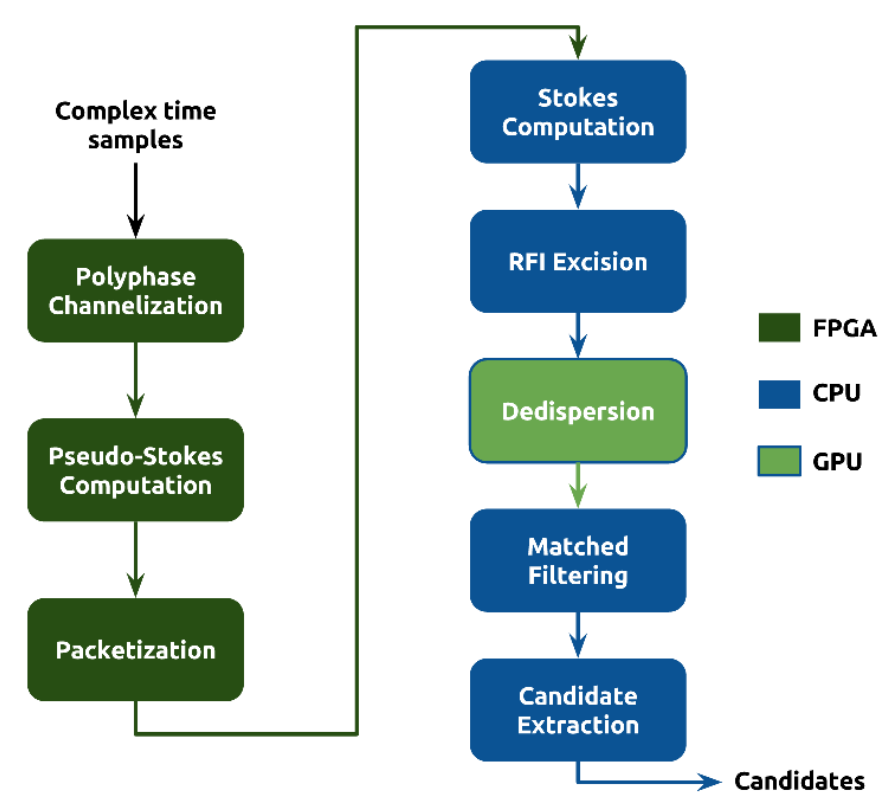

Fig. 1. The signal processing steps in ALFABURST, as described in Sec. 2 The colour of the blocks represents the device the operation takes place in.

equipped with gaming graphics processing units (GPUs). The down-converted radio frequency signal from the receiver is digitized using fast-sampling analog-to-digital converters at the input of the FPGA boards and polyphase-channelized to 4096 channels. 16-bit pseudo-Stokes values $-X X^{*}, Y Y^{*}, \operatorname{Re}\left(X Y^{*}\right)$, and $\operatorname{Im}\left(X Y^{*}\right)$, where $X$ and $Y$ are the Fourier transforms of the two polarizations, and $X^{*}$ and $Y^{*}$ are the respective complex conjugates - are then computed. The data is packetized and sent over 10-Gigabit Ethernet to the HPC cluster.

The HPC cluster is made up of five server-class computers, one of which is the 'head node', and the remaining four are 'compute nodes'. The head node monitors the receiver in use, and if ALFA is enabled, runs the data acquisition programs on the compute nodes. The compute nodes are dual-socket, dual-GPU systems equipped with NVIDIA GeForce GTX TITAN cards and RAID data disks. Each node, barring the last, runs two instances of the software pipeline, each instance handling a single ALFA beam. The last compute node handles the seventh beam.

The realtime pipeline first computes Stokes values from the pseudo-Stokes data, and the total power is used for downstream processing. An adaptive-thresholding RFI excision algorithm removes spurious noise and normalizes the data to have zero 
mean and uniform RMS. This is followed by dedispersion, matched filtering, and thresholding for candidate extraction. The dedispersion stage is based on the GPUbased AstroAccelerate dedispersion transform algorithm. ${ }^{1}$ Fig. 1 shows a schematic of the signal processing operations of the instrument.

We performed commissioning tests of the system between March and July 2015 (for details, see Rajwade et al., these Proceedings). We observed a few pulsars known for their strong single pulses using each of the seven ALFA beams, and were able to blindly detect them using our pipeline. Fig. 2 shows detections from the pulsar B0611+22.

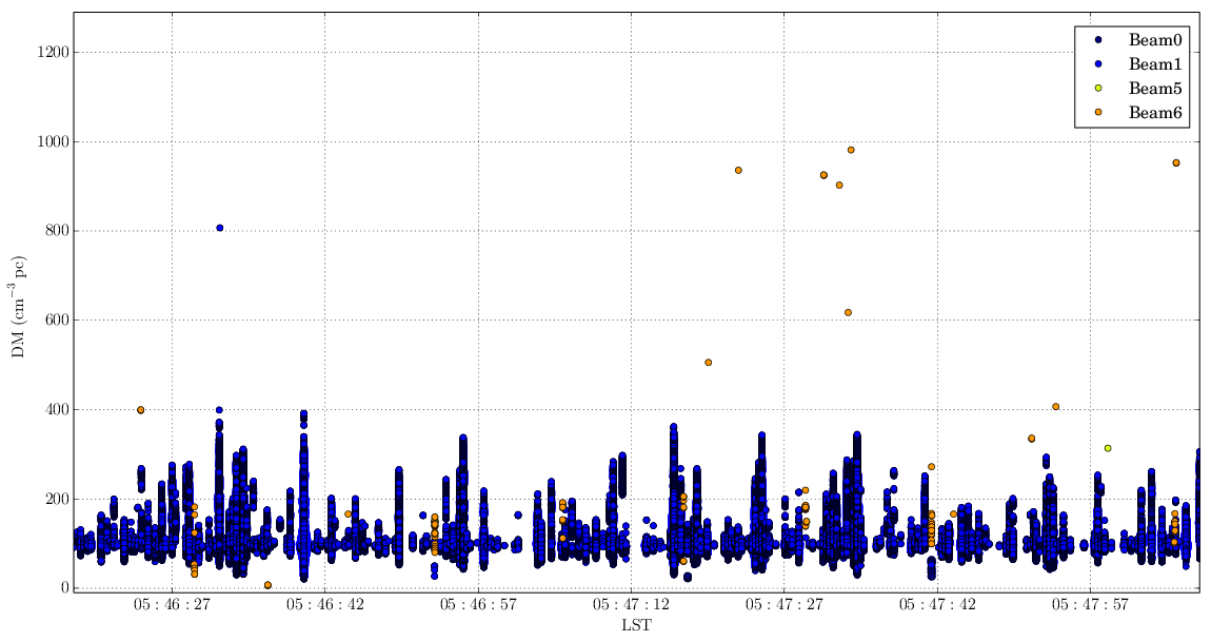

Fig. 2. Results of a commissioning test of the system wherein we observed the pulsar B0611+22 using beam 1 of ALFA, with the pipeline processing the data in realtime. Each marker represents an event that crossed a 6 -sigma threshold. The pulsar $S / N$ peaks around the expected DM of $97 \mathrm{~cm}^{-3} \mathrm{pc}$.

\section{Commensal Survey}

For the commensal survey that we plan to conduct, observations are triggered automatically whenever the primary observer enables the ALFA receiver. Based on historical and expected usage of ALFA, we expect to observe for 500-1000 hours over the next year. Using an event rate of $3.1 \times 10^{4} \mathrm{sky}^{-1}$ day $^{-1}$ above $350 \mathrm{mJy}$ that is based on the ALFA FRB discovery of Ref. 11, an instantaneous field of view of 0.109 sq. deg. that takes into account sensitive sidelobes, and the expected usage of the receiver, we expect to detect $2-3$ FRBs over the next year. 


\section{Acknowledgments}

We thank Dana Whitlow, Arun Venkataraman, Mike Nolan, Phil Perillat and Luis Quintero at the Arecibo Observatory for their assistance during instrument deployment and test observations. JC, AK and WA would like to thank the Leverhulme Trust for supporting this work.

\section{References}

1. W. Armour, et al., A GPU-based Survey for Millisecond Radio Transients Using ARTEMIS, in Astronomical Data Analysis Software and Systems XXI, eds. P. Ballester, D. Egret, N. P. F. Lorente, Vol. 461 (Astron. Soc. Pac. Conf. Series, 2012), pp. 33-36.

2. J. M. Cordes, et al., Arecibo Pulsar Survey Using ALFA. I. Survey Strategy and First Discoveries, Astrophys. J., 637, 446, 2006.

3. H. Falcke and L. Rezzolla, Fast radio bursts: the last sign of supramassive neutron stars, Astron. Astrophys., 562, A137, 2014.

4. A. Karastergiou, et al., Limits on Fast Radio Bursts at $145 \mathrm{MHz}$ with ARTEMIS, a real-time software backend, arXiv:1506.03370, 2015.

5. A. Loeb, Y. Shvartzvald and D. Maoz, Mon. Not. R. Astron. Soc., 439, L46, 2014.

6. D. R. Lorimer, et al., A Bright Millisecond Radio Burst of Extragalactic Origin, Sci., 318, 777, 2007.

7. M. McQuinn, Astrophys. J., 780, L33, 2014.

8. F. Mottez and P. Zarka, Astron. Astrophys., 569, A86, 2014.

9. M. Persic and P. Salucci, Mon. Not. R. Astron. Soc., 258, 14P, 1992.

10. E. Petroff, et al., A real-time fast radio burst: polarization detection and multiwavelength follow-up, Mon. Not. R. Astron. Soc., 447, 246, 2015.

11. L. G. Spitler, et al., Fast Radio Burst Discovered in the Arecibo Pulsar ALFA Survey, Astrophys. J., 790, 101, 2014.

12. D. Thornton, et al., A Population of Fast Radio Bursts at Cosmological Distances, Sci., 341, 53, 2013. 